Trauma Berufskrankh $2006 \cdot 8$ [Suppl 1]: S55-S57 DOI 10.1007/s10039-006-1120-4

Online publiziert: 06. April 2006

๑) Springer Medizin Verlag 2006

R. Simon

BG-Unfallklinik Ludwigshafen, Ludwigshafen

\title{
MRT in der Unfallchirurgie
} Beantwortbare Fragen

der Blutgefäße, Herzschrittmacher, bestimmte Metallimplantate (Osteosynthesematerial), Schwangerschaft oder Klaustrophobie anzuführen. Bei möglichen Kontraindikationen muss im Einzelfall durch den Radiologen geklärt und entschieden werden, ob eine MRT-Untersuchung durchgeführt werden kann. Anzumerken ist, dass bei Patienten mit einliegendem Osteosynthesematerial unter bestimmten Voraussetzungen in vielen Fällen eine MRT-Untersuchung möglich ist.

Im Folgenden werden anhand des Indikationsspektrums beispielhaft typische unfallchirurgische Befunde besprochen.

\section{Typische unfallchirurgische Befunde}

\section{Frisches Trauma}

\section{Knöcherne Verletzung}

Mit der Magnetresonanztomographie können zuverlässig Knochenkontusionen bzw. röntgenologisch okkulte Frakturen (Mikrofrakturen) dargestellt werden. Weiterhin lassen sich andere Stressreaktionen des Knochens in Form eines Knochenmarködems erkennen. Hervorzuheben ist die Diagnostik kindlicher Frakturen ohne Einsatz von Röntgenstrahlen. In der täglichen Routine werden auch immer wieder ,zufällig" Frakturen entdeckt, die im primären Röntgenbild (auch retrospektiv) nicht zu sehen sind. Bei Frakturen im Bereich der Wirbelsäule und der Gelenke können mit der MRT wichtige Begleitverletzungen im Bereich der paraossären und periartikulären Weichteilstrukturen diagnostiziert werden.

\section{Weichteilverletzung}

Mit der MRT ist es möglich, nichtinvasiv Weichteilstrukturen des Stütz- und Bewegungsapparats in hervorragender Qualität darzustellen. Beispiele hierfür sind Bandverletzungen im Bereich aller Gelenkabschnitte sowie Muskel- und Sehnenverletzungen im Bereich der Gelenke und Extremitäten. Hervorzuheben ist der Einsatz der MRT bei komplexen Gelenkverletzungen, z. B. im Bereich des Kniegelenks mit Beteiligung der Gelenkkapsel, der ligamentären Strukturen und Menisken.

Im Bereich der Wirbelsäule können neben Frakturen auch diskoligamentäre Verletzungen und Einblutungen in den Spinalkanal diagnostiziert werden.

\section{Subakutes "chronisches" Trauma und Traumafolgen}

Eine häufige Indikation zur MRT sind ältere Wirbelfrakturen, bei denen sichere Aussagen über die Frakturgeometrie und eine Beteiligung des Spinalkanals gemacht werden können. Mit der Computertomographie können zwar u. U. die knöchernen Verhältnisse morphologisch noch exakter dargestellt werden, eine optimale Beurteilung der paraossären Weichteilstrukturen und insbesondere des Spinalkanalinhalts ist jedoch im CT in gleicher Qualität nicht möglich.

Weitere ältere posttraumatische $\mathrm{Zu}$ stände sind beispielsweise posttraumatische Sekundär- und arthrotische Gelenkveränderungen, bei denen im MRT Aussagen zur Knorpelbeschaffenheit gemacht werden können.

Auch postoperative Zustände, z. B. nach vorderer Kreuzbandersatzplastik, können in der MRT gut dargestellt wer-
Hier sind beispielsweise ferromagnetische Metallclips im Bereich des Gehirns und 
Trauma Berufskrankh 2006 • 8 [Suppl 1]: S55-S57 DOI 10.1007/s10039-006-1120-4

(c) Springer Medizin Verlag 2006

\section{R. Simon}

\section{MRT in der Unfallchirurgie. Beantwortbare Fragen}

\section{Zusammenfassung}

Das Indikationsspektrum der Magnetresonanztomographie (MRT), einem diagnostischen bildgebenden Verfahren ohne Anwendung ionisierender Strahlen, für den Bereich der Unfallchirurgie ist vielfältig und deckt nahezu alle unfallchirurgischen Fragestellungen ab. Bei möglichen Kontraindikationen muss im Einzelfall durch den Radiologen geklärt und entschieden werden, ob eine MRT-Untersuchung durchgeführt werden kann. Im vorliegenden Beitrag werden typische unfallchirurgische Befunde erläutert. So wird im Rahmen des frischen Traumas auf die Möglichkeiten der MRT bei knöchernen und Weichteilverletzungen eingegan-

\section{MRI in trauma surgery. Questions that do have answers}

\begin{abstract}
The range of indications for magnetic resonance imaging (MRI), a diagnostic imaging technique that does not use ionizing radiation, in trauma surgery is wide and covers almost all problems encountered in trauma surgery. When there are any contraindications the radiologist should explain these and decide whether an MRI examination can be carried out nonetheless. In this paper findings typically encountered in trauma surgery are discussed. Within the discussion of fresh trauma, for instance, detailed information on what can be learned from MRI when there are bony and soft tissue injuries is given. Its use in subacute "chronic" trauma, to ex-
\end{abstract}

gen. Auch ihr Einsatz beim subakuten „chronischen" Trauma, zur Abklärung von Traumafolgen und bei entzündlichen Veränderungen wird dargestellt. Differenzialdiagnostisch kommt sie bei unklaren klinischen Befunden und Beschwerden zum Einsatz. Eine weitere wichtige Indikation ist die Abklärung von Traumafolgen gegenüber unfallunabhängigen Veränderungen. Abschließend wird auf spezielle MR-Techniken eingegangen.

\section{Schlüsselwörter}

MRT-Indikationsspektrum • Frisches Trauma • Subakutes „chronisches" Trauma · Entzündliche Veränderungen · Gutachten den. Hier ist jedoch anzumerken, dass nach VKB-Ersatzplastik das Transplantat im Verlauf von mehreren Monaten bis zu 2 Jahren aufgrund von Vaskularisationsund Reparationsvorgängen sehr unterschiedlich zur Darstellung kommt. Dies muss bei der qualitativen Bewertung des Bandersatzes berücksichtigt werden.

\section{Entzündliche Veränderungen}

Mit der Magnetresonanztomographie können nach Gabe von paramagnetischem Kontrastmittel entzündliche Prozesse im Bereich der knöchernen Strukturen und Weichteile in hervorragender Qualität dargestellt werden. Beispiele hierfür sind entzündliche Veränderungen im Bereich der Extremitäten (Osteitis, Osteomyelitis) und der Wirbelsäule (Spondylitis, Spondylodiszitis).

\section{Differenzialdiagnosen bei unklaren klinischen Befunden und Beschwerden} in the case of inflammatory alterations is also presented. This procedure can also be used for differential diagnosis when clinical findings and symptoms are equivocal. It is also indicated when it is difficult to differentiate between late consequences of trauma and accident-dependent changes. Finally, specific MR techniques are discussed in detail.

\section{Keywords}

Range of indications for MRI · Fresh trauma . Subacute "chronic" trauma · Inflammatory changes - Expert's report
Beispiele sind die „zufällige“ Detektion von Tumoren im Bereich des Stützund Bewegungsapparats, wenn aufgrund persistierender Beschwerden, z. B. nach einem Bagatelltrauma, zur weiteren $\mathrm{Ab}$ klärung eine MR-Untersuchung durchgeführt wird. Auch bei einem klinisch und röntgenologisch vermuteten entzündlichen Prozess im Bereich der Wirbelsäule (Spondylitis) kann differenzialdiagnostisch eine Tumorerkrankung vorliegen, die erst mit der MRT diagnostiziert wird.

\section{Abklärung von Traumafolgen/Gutachten}

Der bei vielen (älteren) Patienten relativ häufig diagnostizierte Meniskusschaden im Bereich des Kniegelenks kann mit einer standardisierten Stadieneinteilen exakt klassifiziert werden. In Kenntnis der genauen Anamnese und des klinischen Befunds kann dann in vielen Fällen eine relativ sichere Aussage gemacht werden, ob ein degenerativer oder ein traumatischer Meniskusschaden vorliegt.

Ähnliches gilt für den degenerativen Bandscheibenschaden im Bereich der Wirbelsäule in Abgrenzung zu einer diskoligamentären Verletzung - insbeson- 
dere dann, wenn die MRT-Untersuchung kurze Zeit nach einem entsprechenden Unfallereignis durchgeführt wurde.

Als drittes Beispiel sei der Rotatorenmanschettenschaden im Bereich der Schulter aufgeführt, auch hier können zusammen mit exakten anamnestischen und klinischen Daten oft sichere Aussagen zum Unfallzusammenhang gemacht werden.

\section{Spezielle MR-Techniken}

Hier sind für den Bereich der Unfallchirurgie insbesondere die nichtinvasive MRAngiographie und MR-Myelographie zu erwähnen.

Mit der MR-Angiographie im Bereich der Becken- und Beinarterien kann vor geplanten rekonstruktiven Eingriffen die arterielle Gefäßversorgung exakt dargestellt werden. Eine weitere Indikation im Bereich der Kopf- und Halsarterien sind beispielsweise HWS-Verletzungen mit Gefäßbeteiligung (Dissektion).

Ein Beispiel für die Anwendung der MR-Myelographie ist die (z. B. posttraumatische) Spinalkanalstenose zur Operationsplanung.

\section{Fazit für die Praxis}

Die Magnetresonanztomographie ist ein seit vielen Jahren etabliertes bildgebendes Verfahren, mit dem die gesamte Indikationsbreite der unfallchirurgischen Diagnostik abgedeckt wird.

Für ihren optimalen Einsatz als alleiniges diagnostisches Verfahren oder in Ergänzung zu anderen bildgebenden Modalitäten (Röntgen, $\mathrm{CT}$ ) ist der ständige Dialog zwischen Unfallchirurgen und Radiologen von herausragender Bedeutung.

\section{Korrespondierender Autor}

\section{Dr. R. Simon}

BG-Unfallklinik Ludwigshafen

Ludwig-Guttmann-Straße 13,

67071 Ludwigshafen

simon@bgu-ludwigshafen.de

Interessenkonflikt. Es besteht kein Interessenkonflikt. Der korrespondierende Autor versichert, dass keine Verbindungen mit einer Firma, deren Produkt in dem Artikel genannt ist, oder einer Firma, die ein Konkurrenzprodukt vertreibt, bestehen. Die Präsentation des Themas ist unabhängig und die Darstellung der Inhalte produktneutral. 